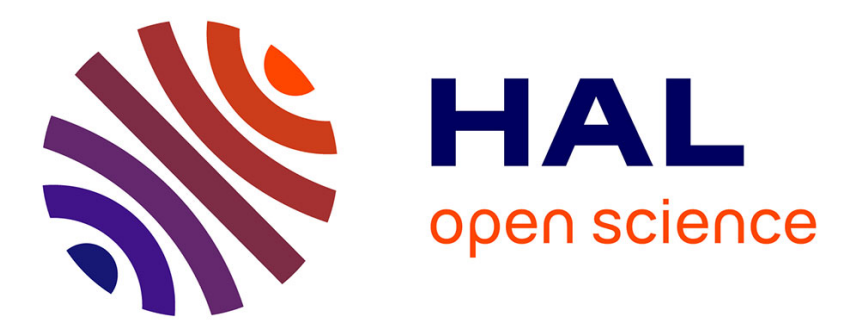

\title{
Variable-order fractional numerical differentiation for noisy signals by wavelet denoising
} Yi-Ming Chen, Yan-Qiao Wei, Da-Yan Liu, Driss Boutat, Xiu-Kai Chen

\section{To cite this version:}

Yi-Ming Chen, Yan-Qiao Wei, Da-Yan Liu, Driss Boutat, Xiu-Kai Chen. Variable-order fractional numerical differentiation for noisy signals by wavelet denoising. Journal of Computational Physics, 2016, 311, pp.338-347. 10.1016/j.jcp.2016.02.013 . hal-02884938

\section{HAL Id: hal-02884938 \\ https://hal.science/hal-02884938}

Submitted on 30 Jun 2020

HAL is a multi-disciplinary open access archive for the deposit and dissemination of scientific research documents, whether they are published or not. The documents may come from teaching and research institutions in France or abroad, or from public or private research centers.
L'archive ouverte pluridisciplinaire HAL, est destinée au dépôt et à la diffusion de documents scientifiques de niveau recherche, publiés ou non, émanant des établissements d'enseignement et de recherche français ou étrangers, des laboratoires publics ou privés. 


\title{
Variable-order fractional numerical differentiation for noisy signals by wavelet denoising
}

\author{
Yi-Ming Chen ${ }^{a}$, Yan-Qiao Wei ${ }^{\mathrm{a}, *}$, Da-Yan Liu ${ }^{\mathrm{b}}$, Driss Boutat ${ }^{\mathrm{b}}$, Xiu-Kai Chen ${ }^{\mathrm{a}}$ \\ ${ }^{a}$ College of Sciences, Yanshan University, Qinhuangdao, 066004, Hebei, China \\ ${ }^{\mathrm{b}}$ INSA Centre Val de Loire, Université d'Orléans, PRISME EA 4229, 18022 Bourges, France
}

\begin{abstract}
In this paper, an efficient method is proposed to numerically estimate the variable-order fractional derivatives of a noisy signal. Firstly, the process of wavelet denoising is adopted to reduce the noise effect in the signal. Secondly, polynomials are constructed to fit the denoised signal in a set of overlapped subintervals of the considered interval. Thirdly, the variable-order fractional derivatives of these fitting polynomials are considered as the estimations of the original signal, where the values obtained near the boundaries of each subinterval are ignored in the overlapped parts. Finally, numerical examples are presented to demonstrate the efficiency and robustness of the proposed method.
\end{abstract}

Keywords: Variable-order fractional numerical differentiation; Noisy signals; Wavelet denoising; Fitting polynomials;

\section{Introduction}

In recent decades, fractional calculus has been successfully extended from pure mathematical theory to practical applications, such as visco-elastic materials $[1,2]$, economics [3], statistical mechanics [4], as well as solid mechanics [5], etc. Meanwhile, the booming development of numerical methods focusing on the computing difficulties for fractional calculus promotes the topic to go further. These methods include finite difference method [6], Laplace transform method[7], Adomian decomposition method [8], variational iteration method [9], fractional differential transform method [10], operational approach [11, 12], as well as orthogonal functions methods including Block pulse functions [13], Bernstein polynomials [14], generalized fractional-order Legendre functions [15], Chebyshev wavelets [16], Legendre wavelets [17], etc.

Fractional order numerical differentiation in signal processing is a relevant research branch. Numerous effective numerical algorithms have been proposed by researchers [18-27]. With regard to a noisy signal, a filter will generally be used to smooth the signal and then the fractional derivative of the filter is considered as a differentiator. In [20], the Digital Fractional Order Savitzkv-Golav Differentiator (DFOSGD) was shown to be better than some other existing fractional order differentiators. Afterwards, the fractional order Jacobi differentiator was presented and shown better performance than the DFOSGD both in noisy and noise-free cases [21, 22]. In [23], a method combining B-Spline functions with Tikhonov regularization was proposed.

Currently, the topic of variable-order fractional calculus is becoming more and more attractive. A variety of works have been presented [28-37]. In [28-30], the authors have built

\footnotetext{
* Corresponding author. Tel: +8615512061089.

E-mail address: weiyanqiao2014@163.com (Y.W)
} 
several mathematical models by applying the variable-order fractional calculus. Due to the existence of variable-order fractional differential and integral operators, the solutions for such kind of problems were always quite difficult to find. Thus, it is necessary to develop efficient numerical techniques. In [29, 30], numerical methods for variable-order fractional differential equations based on finite difference method were presented. In [33, 34], Chen and Liu et al. provided a distinctive class of numerical method based on operational matrices of Bernstein polynomials for variable-order fractional linear cable equation and time fractional diffusion equation respectively. Legendre wavelets functions were also adopted to numerically solve similar problems in [35]. Zayernouri and Karniadakis applied fractional spectral collocation methods for linear and nonlinear variable-order FPDEs in [36]. Moreover, Zhao et al. proposed second-order approximations for variable-order fractional derivatives and gave both algorithms and applications in [37]. On account of these works, it is convinced that the relative researches can be fairly promising.

Based on such research background, variable-order fractional numerical differentiation of a noisy signal is considered in this paper. As well known, wavelet method is a prominent tool in signal processing. In this paper, a noisy signal is processed by means of wavelet denoising. Then, fitting polynomials are adopted to approximate the denoised signal via solving least square problems. Thus, the variable-order fractional numerical differentiation can be performed easily.

The outline of this paper is as follows: In Section 2, some necessary mathematical definitions and properties of variable-order fractional differential operators are introduced. In Section 3, the denoising process of wavelet method is presented. In Section 4, the denoised signal is fitted by polynomials and the variable-order fractional numerical differentiation is studied in Section 5. In Section 6, some examples are illustrated. Finally, some conclusions are given in Section 7.

\section{Preliminaries}

This section presents some definitions as well as some properties on fractional calculus and wavelet analysis, which will be useful in this work.

\subsection{Variable-order fractional derivative}

In this paper, variable-order Caputo fractional derivatives are considered, which are defined as follows.

Definition 2.1 [33]: Let $a \in \mathbf{R}$, and $f \in C^{1}(\mathbf{R})$, where $C^{1}(\mathbf{R})$ refers to the set of functions being 1-time continuously differentiable on $\square$. Then, the variable-order Caputo fractional derivative of $f$ is defined as follows:

$$
\forall t>a, \quad D_{a, t}^{\alpha(t)} f(\square)=\frac{1}{\Gamma(1-\alpha(t))} \int_{a}^{t}(t-\tau)^{-\alpha(t)} f^{\prime}(\tau) d \tau,
$$

where $0<\alpha(t) \leq 1$, and $\Gamma(\square)$ is the Gamma function. 
Then, the following two properties can be deduced.

Property 2.1 [33]: Let $f(t)=(t-a)^{n}$, with $0<\alpha(t) \leq 1$ and $a \leq t \in \mathbf{R}$, then:

$$
D_{a, t}^{\alpha(t)}(t-a)^{n}= \begin{cases}\frac{\Gamma(n+1)}{\Gamma(n+1-\alpha(t))}(t-a)^{n-\alpha(t)}, & n \neq 0, \\ 0, & n=0 .\end{cases}
$$

Property 2.2: Let $f_{1}(t) \in C^{1}(\mathbf{R}), f_{2}(t) \in C^{1}(\mathbf{R})$, with $0<\alpha(t) \leq 1$ and $a \leq t \in \mathbf{R}$, then:

$$
D_{a, t}^{\alpha(t)}\left(\lambda_{1} f_{1}(t)+\lambda_{2} f_{2}(t)\right)=\lambda_{1} D_{a, t}^{\alpha(t)} f_{1}(t)+\lambda_{2} D_{a, t}^{\alpha(t)} f_{2}(t)
$$

\subsection{Theory foundation of wavelet analysis}

Definition 2.2 (Frame and Riesz bases) [38]: A sequence $\left\{\phi_{n}\right\}_{n \in \Lambda}$ is called a frame of a Hilbert space $\mathbf{H}$, if there exist two constants $B \geq A>0$ such that

$$
\forall f \in \mathbf{H}, A\|f\|^{2} \leq \sum_{n \in \Lambda}\left|\left\langle f, \phi_{n}\right\rangle\right|^{2} \leq B\|f\|^{2} .
$$

$\Lambda$ refers to a finite or infinite index set. If $A=B$, then the frame is compact. If the frame $\left\{\phi_{n}\right\}_{n \in \Lambda}$ is linear independent, it is irredundant and called Riesz bases.

Based on Definition 2.2, the fundamental concept of "Multiresolution approximation" of wavelet analysis can be introduced as follows.

Definition 2.3 (Multiresolution approximation) [38]: Let $f \in \mathbf{L}^{2}[\mathbf{R}]$. If the following six properties are fulfilled, the sequence of closed subspace $\left\{\mathbf{V}_{j}\right\}_{j \in \mathbf{Z}} \in \mathbf{L}^{2}[\mathbf{R}]$ is called a multiresolution approximation:

$$
\begin{aligned}
& \forall(j, k) \in \mathbf{Z}^{2}, f(t) \in \mathbf{V}_{j} \Leftrightarrow f\left(t-2^{j} k\right) \in \mathbf{V}_{j}, \\
& \forall j \in \mathbf{Z}, \mathbf{V}_{j+1} \subset \mathbf{V}_{j}, \\
& \forall j \in \mathbf{Z}, f(t)=\mathbf{V}_{j} \Leftrightarrow f\left(\frac{t}{2}\right) \in \mathbf{V}_{j+1}, \\
& \lim _{j \rightarrow+\infty} \mathbf{V}_{j}=\overbrace{j=-\infty}^{+\infty} \mathbf{V}_{j}=\{0\}, \\
& \lim _{j->-\infty} \mathbf{V}_{j}=\text { Closure }\left(\bigcup_{j=-\infty}^{+\infty} \mathbf{V}_{j}\right)=\mathbf{L}^{2}[\mathbf{R}],
\end{aligned}
$$

$\{\theta(t-n)\}_{n \in \mathbf{Z}}$ is a group of Riesz bases of $\mathbf{V}_{0}$ if there exists a $\theta$.

\subsection{Problem statement}

Let $b, c \in \mathbf{R}$, and $y \in C^{1}(\mathbf{R})$. Assume that $y^{\omega}\left(t_{i}\right)=y\left(t_{i}\right)+\delta \omega\left(t_{i}\right)$ is a discrete noisy observation of the continuous signal $y(t)$ on $I=[b, c]$, with $t_{i}=b+T_{s} i$, for $i=1,2, \cdots, M$, 
where $T_{s}=\frac{c-b}{M-1}$ is an equidistant sampling period. The noise $\left\{\delta \omega\left(t_{i}\right)\right\}$ is a sequence of random variables with zero-mean and an unknown variance $\delta$. Then, the considered problem is to provide an approach to numerically compute the variable-order fractional derivative $D_{b, t_{i}}^{\alpha\left(t_{i}\right)} y\left(t_{i}\right)$ from the noisy signal $y^{\omega}$.

\section{Main results}

\subsection{The process of wavelet denoising}

In [38], S. Mallat provided a quite systematic introduction on wavelet tour in signal processing. Based on this excellent monograph, some basic theory of wavelet denoising is briefly recalled in this section.

Suppose that a signal $f$ and a sequence of closed subspace $\left\{\mathbf{V}_{j}\right\}_{j \in \mathbf{Z}} \in \mathbf{L}^{2}[\mathbf{R}]$ are given based on Definition 2.2 and Definition 2.3. By means of multiresolution approximation, the signal $f$ can be analyzed in different levels of subspaces $\left\{\mathbf{V}_{j}\right\}_{j \in \mathbf{Z}}$.

The orthogonal projection of $f$ on $\mathbf{V}_{j}$ is given by $P_{\mathbf{v}_{j}} f=\sum_{n=-\infty}^{+\infty}\left\langle f, \phi_{j, n}\right\rangle \phi_{j, n}$, where $\left\{\phi_{j, n}\right\}_{n \in \mathbf{Z}}$ is a set of scale orthogonal bases of $\mathbf{V}_{j}$. The scale bases $\left\{\phi_{j, n}\right\}_{n \in \mathbf{Z}}$ can be obtained by dilating and shifting a single scale function $\phi$ as:

$$
\phi_{j, n}(t)=\frac{1}{\sqrt{2^{j}}} \phi\left(\frac{t-2^{j} n}{2^{j}}\right), n \in \mathbf{Z} .
$$

If $a_{j}[n]=\left\langle f, \phi_{j, n}\right\rangle$ is denoted as the associated inner product, then it can be rewritten into the convolution form as follows:

$$
a_{j}[n]=\int_{-\infty}^{+\infty} f(t) \frac{1}{\sqrt{2^{j}}} \phi\left(\frac{t-2^{j} n}{2^{j}}\right) d t=f * \bar{\phi}_{j}\left(2^{j} n\right) .
$$

Since $V_{j} \subset V_{j-1}$, there exists a set $\mathbf{W}_{j}$ such that

$$
\mathbf{V}_{j-1}=\mathbf{V}_{j} \oplus \mathbf{W}_{j} .
$$

Then, the orthogonal projection of $f$ can be respectively decomposed from $\mathbf{V}_{j-1}$ to its subspaces $\mathbf{V}_{j}$ and $\mathbf{W}_{j}$ as follows:

$$
P_{\mathbf{v}_{j-1}} f=P_{\mathbf{v}_{j}} f+P_{\mathbf{w}_{j}} f .
$$

By dilating and shifting a wavelet $\psi$, orthogonal bases $\left\{\psi_{j, n}\right\}_{n \in \mathbf{Z}}$ of $\mathbf{W}_{j}$ can be obtained as follows:

$$
\psi_{j, n}(t)=\frac{1}{\sqrt{2^{j}}} \psi\left(\frac{t-2^{j} n}{2^{j}}\right), n \in \mathbf{Z} .
$$

Denote $d_{j}[n]=\left\langle f, \psi_{j, n}\right\rangle$. Then, according to Eq. (6), we have: 


$$
f(t)=\sum_{n=-\infty}^{+\infty} a_{j}[n] \phi_{j, n}(t)+\sum_{n=-\infty}^{+\infty} d_{j}[n] \psi_{j, n}(t) .
$$

Let $\bar{x}[n]=x[-n]$ and

$$
\hat{x}[n]= \begin{cases}x[p], & n=2 p, \\ 0, & n=2 p+1,\end{cases}
$$

then we can introduce the following theorem.

Theorem 3.1[39, 40]: Signal decomposing formula:

$$
\begin{aligned}
& a_{j+1}[p]=\sum_{n=-\infty}^{+\infty} h[n-2 p] a_{j}[n]=a_{j} * \bar{h}[2 p], \\
& d_{j+1}[p]=\sum_{n=-\infty}^{+\infty} g[n-2 p] a_{j}[n]=a_{j} * \bar{g}[2 p],
\end{aligned}
$$

where $h[n-2 p]=\left\langle\frac{1}{\sqrt{2}} \phi\left(\frac{t}{2}\right), \phi(t-n+2 p)\right\rangle, g[n-2 p]=\left\langle\frac{1}{\sqrt{2}} \psi\left(\frac{t}{2}\right), \psi(t-n+2 p)\right\rangle$.

Signal reconstructing formula:

$$
a_{j}[p]=\sum_{n=-\infty}^{+\infty} h[p-2 n] a_{j+1}[n]+\sum_{n=-\infty}^{+\infty} g[p-2 n] d_{j+1}[n]=\hat{a}_{j+1} * h[p]+\hat{d}_{j+1} * g[p] .
$$

It's generally in practical applications that the previously considered signal $f$ is known at uniform sampling points and various kinds of elegant wavelets have been widely used, such as Haar wavelet, Meyer wavelet, Daubechies wavelets, Morlet wavelet, Mexican hat wavelet, etc. Meanwhile, the convolution used above is computed via discrete convolution.

By means of Theorem 3.1, the low-pass filter $\bar{h}$ can remove the high-frequency part of the signal while the high-pass filter $\bar{g}$ reserves this part. Besides, according to Eq.(10) and Eq.(11), a signal can be successively decomposed several times. Thus, if all the elements of the sequence $\left\{d_{j+1}[n]\right\}_{n \in \mathbf{Z}}$ obtained by decomposing a noisy signal are set to zero and in order to reconstruct the signal, then the noise effect can be reduced to a certain degree in the original signal.

\subsection{Polynomial fitting for the denoised signal}

By using the wavelet method given in Section 3.2, we can get a signal with less noise effect. In order to obtain a smoother signal with an algebraic expression, the polynomial fitting method is adopted.

Suppose that the denoised signal $f_{d}$ is located on the time domain $I=[b, c]$, then we define a set of overlapped subintervals of $I$ as follows:

$$
\bigcup_{i=0}^{l}\left[b_{i}, c_{i}\right]=[b, c], b_{k}=b_{k-1}+(c-b) /(2 l), c_{k}=b_{k}+(c-b) / l, k=1,2, \ldots, l .
$$

It should be mentioned that neighboring subintervals are intersecting. 
Define $q$ th order fitting polynomial on $I_{i}=\left[b_{i}, c_{i}\right]$, for $i=0, \ldots, l$ :

$$
F_{I_{i}}(t)=w_{i, q}\left(t-b_{i}\right)^{q}+w_{i, q-1}\left(t-b_{i}\right)^{q-1}+\cdots+w_{i, 1}\left(t-b_{i}\right)+w_{i, 0},
$$

where the coefficients vector $\mathbf{X}_{i}=\left[w_{i, p}, w_{i, p-1}, \ldots, w_{i, 0}\right]^{T}$ are to be determined.

Denote the uniform sampling nodes as a vector $\mathbf{T}_{i}=\left[t_{1}, t_{2}, \ldots, t_{m}\right]^{T}$, then we have:

$$
\mathbf{A}_{i} \mathbf{X}_{i}=f_{d}\left(\mathbf{T}_{i}\right)
$$

where

$$
\mathbf{A}_{i}=\left[\begin{array}{ccccc}
\left(t_{1}-b_{i}\right)^{q} & \left(t_{1}-b_{i}\right)^{q-1} & \cdots & \left(t_{1}-b_{i}\right) & 1 \\
\left(t_{2}-b_{i}\right)^{q} & \left(t_{2}-b_{i}\right)^{q-1} & \cdots & \left(t_{2}-b_{i}\right) & 1 \\
\left(t_{3}-b_{i}\right)^{q} & \left(t_{3}-b_{i}\right)^{q-1} & \cdots & \left(t_{3}-b_{i}\right) & 1 \\
\vdots & \vdots & \ddots & \vdots & \vdots \\
\left(t_{m}-b_{i}\right)^{q} & \left(t_{m}-b_{i}\right)^{q-1} & \cdots & \left(t_{m}-b_{i}\right) & 1
\end{array}\right],
$$

and $f_{d}\left(\mathbf{T}_{i}\right)$ denotes a part of the denoised signal $f_{d}$ at nodes $\mathbf{T}_{i}$. The solution of the equation can be obtained via least square method. If the well-known "economy size" QR decomposition is applied to the matrix $\mathbf{A}_{i}$ such that $\mathbf{Q}_{i} \mathbf{R}_{i}=\mathbf{A}_{i}$, where $\mathbf{Q}_{i}$ is a $m \times(q+1)$

matrix, $\quad \mathbf{R}_{i}$ is a $(q+1) \times(q+1)$ upper triangular matrix if $\mathbf{A}_{i}$ has more rows than columns, then the least square solution of Eq. (15) can be obtained by

$$
\mathbf{X}_{i}=\mathbf{R}_{i}^{-1} \mathbf{Q}_{i}^{T} f_{d}\left(\mathbf{T}_{i}\right)
$$

In this way, the fitting polynomials are obtained in each subinterval.

\subsection{Variable-order fractional derivatives of a noisy signal}

In Section 4, the denoised signal is fitted by polynomials in each subinterval. Then, the variable-order fractional numerical differentiation can be computed via Property 2.1. Eventually, results of all subintervals will be merged together for the entire interval.

By applying Property 2.1 to the fitting polynomial of a subinterval $I_{i}$, we have:

$$
\begin{aligned}
D_{a, t}^{\alpha(t)} F_{I_{i}}(t) & =w_{i, q} \frac{\Gamma(q+1)}{\Gamma(q+1-\alpha(t))}\left(t-b_{i}\right)^{(q-\alpha(t))}+w_{i, q-1} \frac{\Gamma(q)}{\Gamma(q-\alpha(t))}\left(t-b_{i}\right)^{(q-1-\alpha(t))}+\cdots \\
& +w_{i, 1} \frac{\Gamma(2)}{\Gamma(2-\alpha(t))}\left(t-b_{i}\right)^{(1-\alpha(t))}+w_{i, 0} \frac{\Gamma(1)}{\Gamma(1-\alpha(t))}\left(t-b_{i}\right)^{(-\alpha(t))} .
\end{aligned}
$$

Then the variable-order fractional derivatives at each node of subinterval $I_{i}$ can be computed. In order to eliminate results with excessive numerical errors, we merge together the results of all subintervals in the following way: 
By taking subintervals $I_{i}=\left[b_{i}, c_{i}\right]$ and $I_{i+1}=\left[b_{i+1}, c_{i+1}\right]$ as an example, we reserve the part $\left[b_{i},\left(b_{i+1}+c_{i}\right) / 2\right]$ of $I_{i}$ and the part $\left[\left(b_{i+1}+c_{i}\right) / 2, c_{i+1}\right]$ of $I_{i+1}$.

Similarly, executing this procedure to all subspaces one by one can reduce the excessive numerical errors appearing on the boundaries of each subinterval to a certain degree.

\section{Numerical examples}

In this section, some numerical examples are given to show the efficiency and the robustness of the proposed wavelet method. In order to have a forceful verification of the method, a numerical approximate scheme of the variable-order Caputo fractional derivative of a noise-free signal $f \in C^{1}(\mathbf{R})$ located in interval $I=[b, c] \quad\left(b=t_{1}<t_{2} \cdots<t_{i}<\cdots<t_{M}=c\right.$, $\left.h_{i}=t_{i+1}-t_{i}\right)$ is provided based on Ref. [32] as follow:

$$
\begin{aligned}
D_{b, t}^{\alpha\left(t_{i}\right)} f\left(t_{i}\right) & =\frac{1}{\Gamma\left(1-\alpha\left(t_{i}\right)\right)} \int_{b}^{t_{i}}\left(t_{i}-\sigma\right)^{-\alpha\left(t_{i}\right)} f^{\prime}(\sigma) d \sigma \quad\left(i>1 \text { and } D_{b, t}^{\alpha\left(t_{1}\right)} f\left(t_{1}\right)=0\right) \\
& =\frac{1}{\Gamma\left(1-\alpha\left(t_{i}\right)\right)} \sum_{j=1}^{i-1} \int_{t_{j}}^{t_{j+1}}\left(t_{i}-\sigma\right)^{-\alpha\left(t_{i}\right)} f^{\prime}(\sigma) d \sigma \\
& =\frac{1}{\Gamma\left(1-\alpha\left(t_{i}\right)\right)} \sum_{j=1}^{i-1} \int_{t_{j}}^{t_{j+1}}\left(t_{i}-\sigma\right)^{-\alpha\left(t_{i}\right)}\left(\frac{f\left(t_{j+1}\right)-f\left(t_{j}\right)}{h_{j}}+C_{i j} h_{j}\right) d \sigma \\
& =\frac{1}{\Gamma\left(1-\alpha\left(t_{i}\right)\right)} \sum_{j=1}^{i-1}\left(\frac{f\left(t_{j+1}\right)-f\left(t_{j}\right)}{h_{j}}\right) \int_{t_{j}}^{t_{j+1}}\left(t_{i}-\sigma\right)^{-\alpha\left(t_{i}\right)} d \sigma+r_{i} \\
& =\frac{1}{\Gamma\left(2-\alpha\left(t_{i}\right)\right)} \sum_{j=1}^{i-1}\left(\frac{f\left(t_{j+1}\right)-f\left(t_{j}\right)}{h_{j}}\right)\left[\left(t_{i}-t_{j}\right)^{1-\alpha\left(t_{i}\right)}-\left(t_{i}-t_{j+1}\right)^{1-\alpha\left(t_{i}\right)}\right]+r_{i},
\end{aligned}
$$

where the residue $r_{i}$ has

$$
\begin{aligned}
r_{i} & =\frac{1}{\Gamma\left(1-\alpha\left(t_{i}\right)\right)} \sum_{j=1}^{i-1} \int_{t_{j}}^{t_{j+1}}\left(t_{i}-\sigma\right)^{-\alpha\left(t_{i}\right)} C_{i j} h_{j} d \sigma \\
& =\frac{1}{\Gamma\left(2-\alpha\left(t_{i}\right)\right)} \sum_{j=1}^{i-1} C_{i j} h_{j}\left[\left(t_{i}-t_{j}\right)^{1-\alpha\left(t_{i}\right)}-\left(t_{i}-t_{j+1}\right)^{1-\alpha\left(t_{i}\right)}\right] \\
& \leq \frac{\max _{1 \leq j \leq i-1} C_{i j}\left(\max _{1 \leq j \leq i-1} h_{j}\right)^{2-\alpha(t)}}{\Gamma\left(2-\alpha\left(t_{i}\right)\right)} \sum_{j=1}^{i-1}\left[(i-j)^{1-\alpha\left(t_{i}\right)}-(i-j-1)^{1-\alpha\left(t_{i}\right)}\right] \\
& \leq \frac{(M-1)^{1-\alpha\left(t_{i}\right)} \max _{1 \leq j \leq i-1} C_{i j}\left(\max h_{j}\right)^{2-\alpha(t)}}{\Gamma\left(2-\alpha\left(t_{i}\right)\right)} .
\end{aligned}
$$

Additionally, in order to provide an apparent comparison with the proposed method, the mean value of estimation errors is given as follows:

$$
E_{I^{\varepsilon}}=\frac{1}{K} \sum_{i=1}^{K}\left|e_{D}\left(t_{N_{i}}\right)\right|^{2}
$$


where $I^{\varepsilon}=[b-\varepsilon, c-\varepsilon]=\left[t_{N_{1}}, t_{N_{K}}\right], \varepsilon>0$ and $e_{D}$ is the estimation error of the variableorder fractional derivative at each node.

The wavelet method is accomplished via the powerful software MATLAB R2012a, where discrete Meyer wavelet included in the Wavelet toolbox is adopted to decompose a signal to the forth level. Meanwhile, 8-order polynomials are used on each subinterval to fit the denoised signals.

Example 4.1(Periodic signal) Let $y(t)=\sin (5 t)$. Take $I=[0,6], \quad M=5001$, $\alpha(t)=|\cos (5 t)|$ and the length of each subinterval of $I$ is 1.2. The variance $\delta$ is chosen such that the Signal-to-Noise Ratio $S N R=10 \log _{10}\left(\frac{\sum\left|y^{\omega}\left(t_{i}\right)\right|^{2}}{\sum\left|\delta \omega\left(t_{i}\right)\right|_{2}^{2}}\right)$ is equal to $10 \mathrm{~dB}$. The variable-order Caputo fractional derivative of $\sin (w t)$ can be obtained by means of the software Mathematica 9.0. The original noisy signal is shown in Fig 4.1(a) and the denoised signal is shown in Fig 4.1 (b). The estimated variable-order fractional derivative is depicted in Fig 4.2.

Take $\varepsilon=0.0120$ in Eq. (20) and the corresponding values for Eq. (19) and the wavelet method are 5.9724e-07 and 0.0138 respectively. Accordingly, it can be concluded that Eq. (19) can efficiently approximate the exact variable-order Caputo fractional derivative of a noise-free signal and the wavelet method performs well in estimating the variable-order fractional derivative of a noisy periodic signal.

(a)

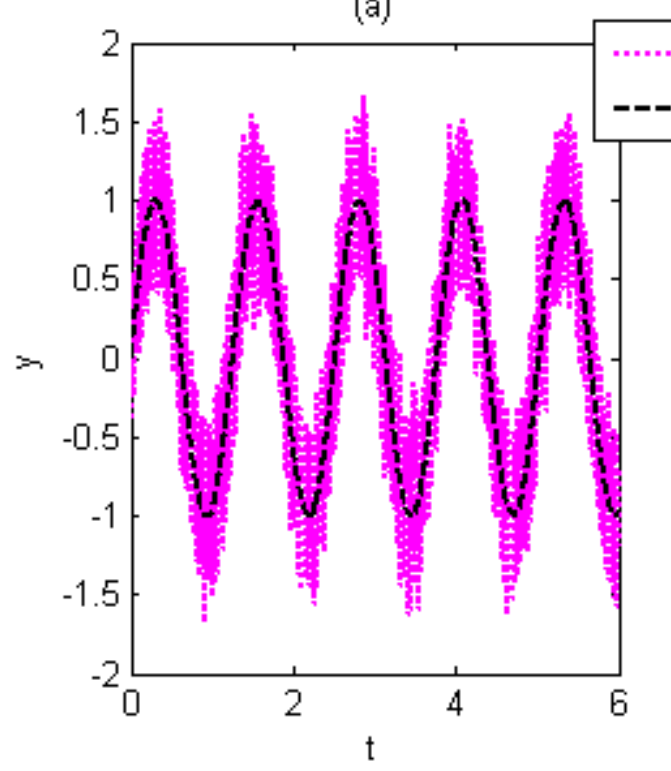

(b)

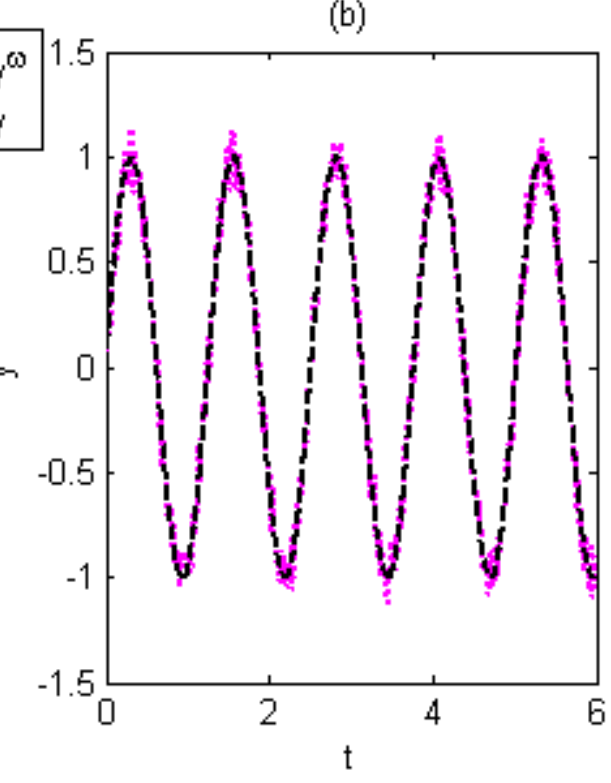

Fig 4.1 Denoising result of the signal in Example 4.1 


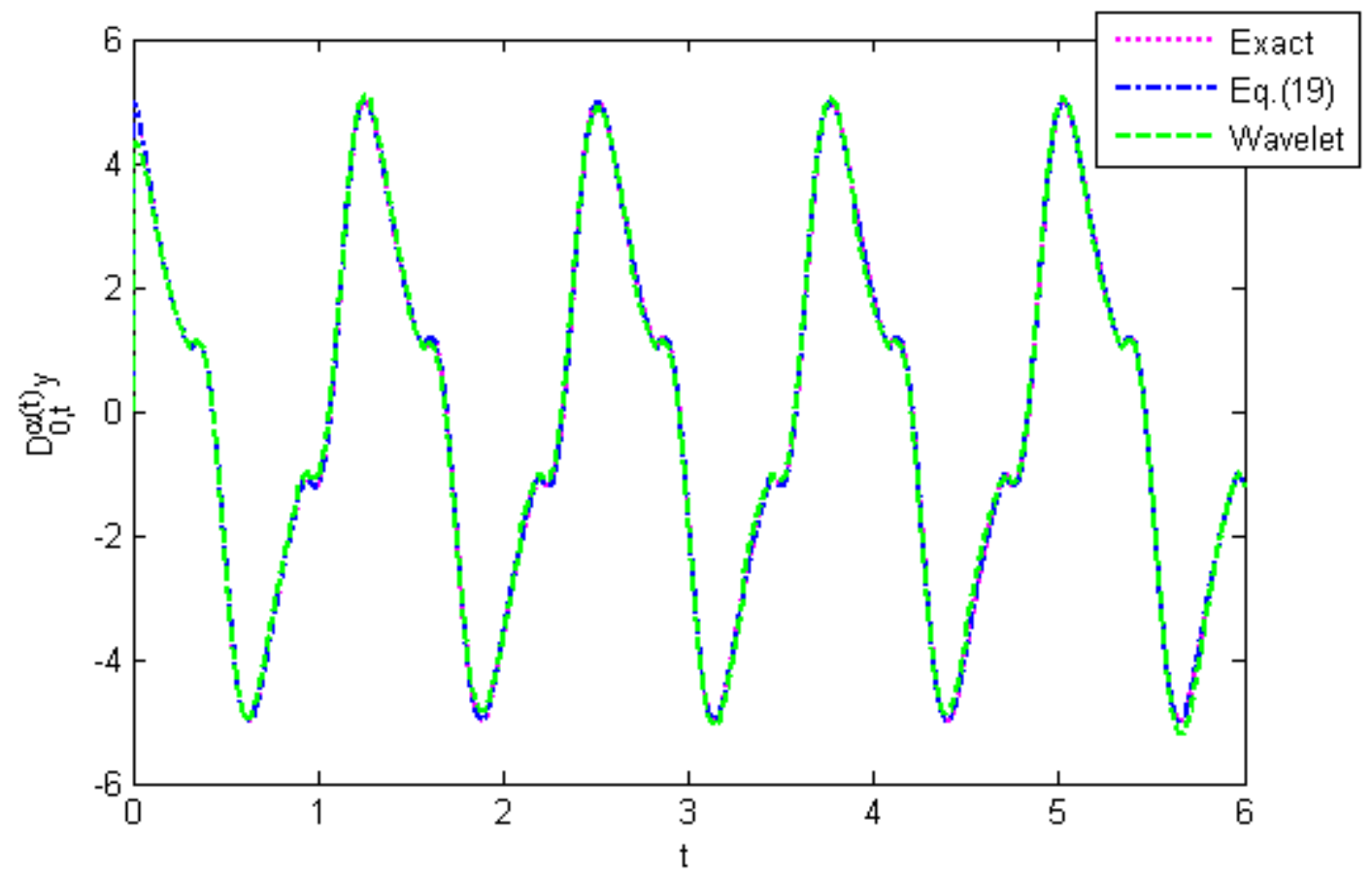

Fig 4.2 The estimated variable-order fractional derivative of Example 4.1

Example 4.2 (Non-periodic signal) Let $y(t)=0.01(t-6)^{2} \sin (6 t)$. Take $I=[0,10]$, $M=10001, \alpha(t)=\left|\cos \left(t^{2}+3 t\right)\right|$ and the length of each subinterval of $I$ is 1 . The variance $\delta^{2}$ is chosen such that the Signal-to-Noise Ratio $S N R=10 d B$. The original noisy signal is shown in Fig 4.3(a) and the denoised signal is shown in Fig 4.3 (b). The estimated variable-order fractional derivative based on Eq. (18) is depicted in Fig 4.4. Take $\varepsilon=0.01$ in Eq. (20), the corresponding result between Eq. (19) and the wavelet method is 3.5351e-04. The validity of the wavelet method for non-periodic signal is shown.

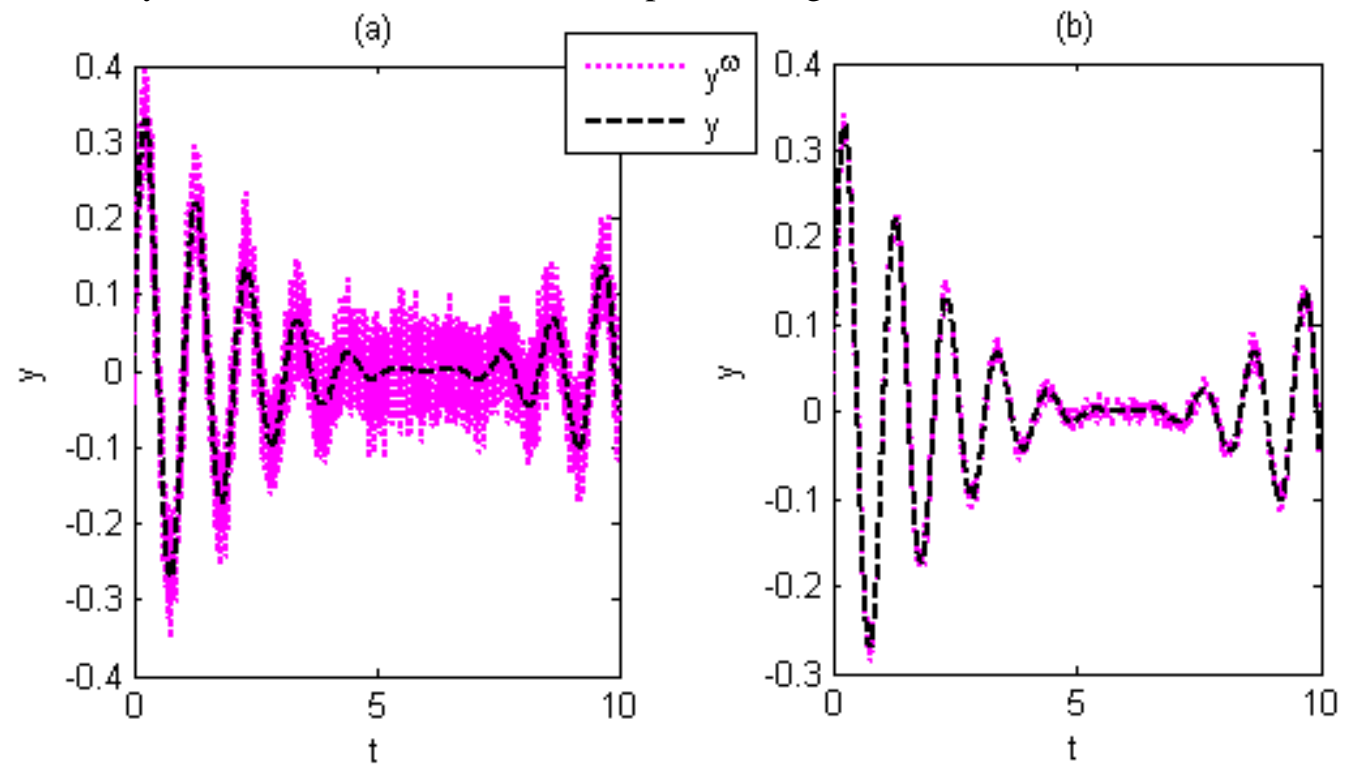

Fig 4.3 Denoising result of the signal in Example 4.2 


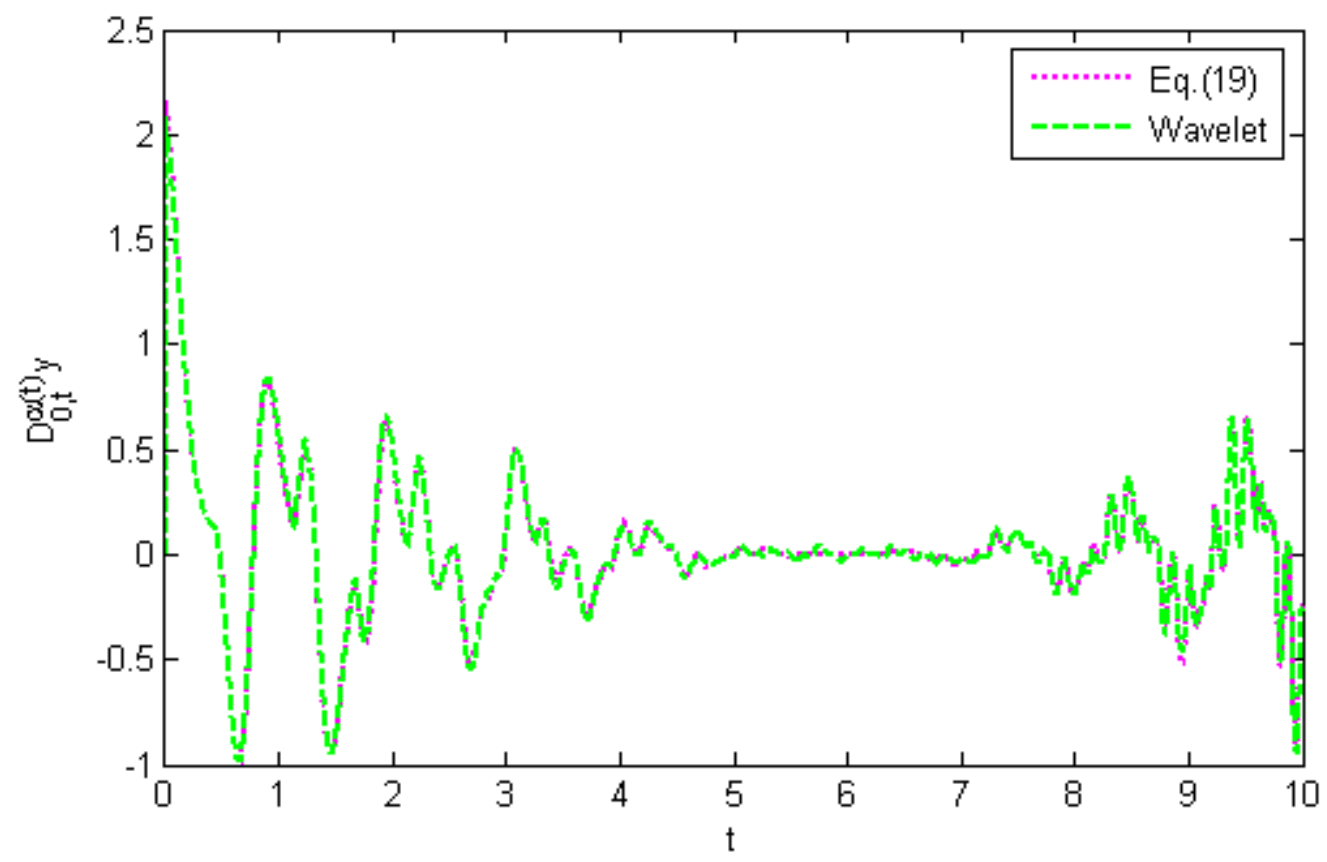

Fig 4.4 The estimated variable-order fractional derivative of Example 4.2

Example 4.3 (Non-equidistant sampling signal) Let $y(t)=\ln (t+1)(\sin (3 t)+\cos (5 t))$.

Take $\alpha(t)=|0.75 \sin (0.25 t)| \quad, \quad M=15001$ and non-equidistant sampling steps $h_{i}=0.001\left(1+\Delta_{i}\right), \Delta_{i} \in[-0.1,0.1], i=1,2, \ldots M-1$. Chose $\delta=0.9$ then $S N R=7.9534 d B$. In order to make the proposed method be applicable in this case, the length of each subinterval can be modified as $l_{i}=1+0.001 \sum_{t_{j} \in I_{i}} \Delta_{j}$. The original noisy signal is shown in

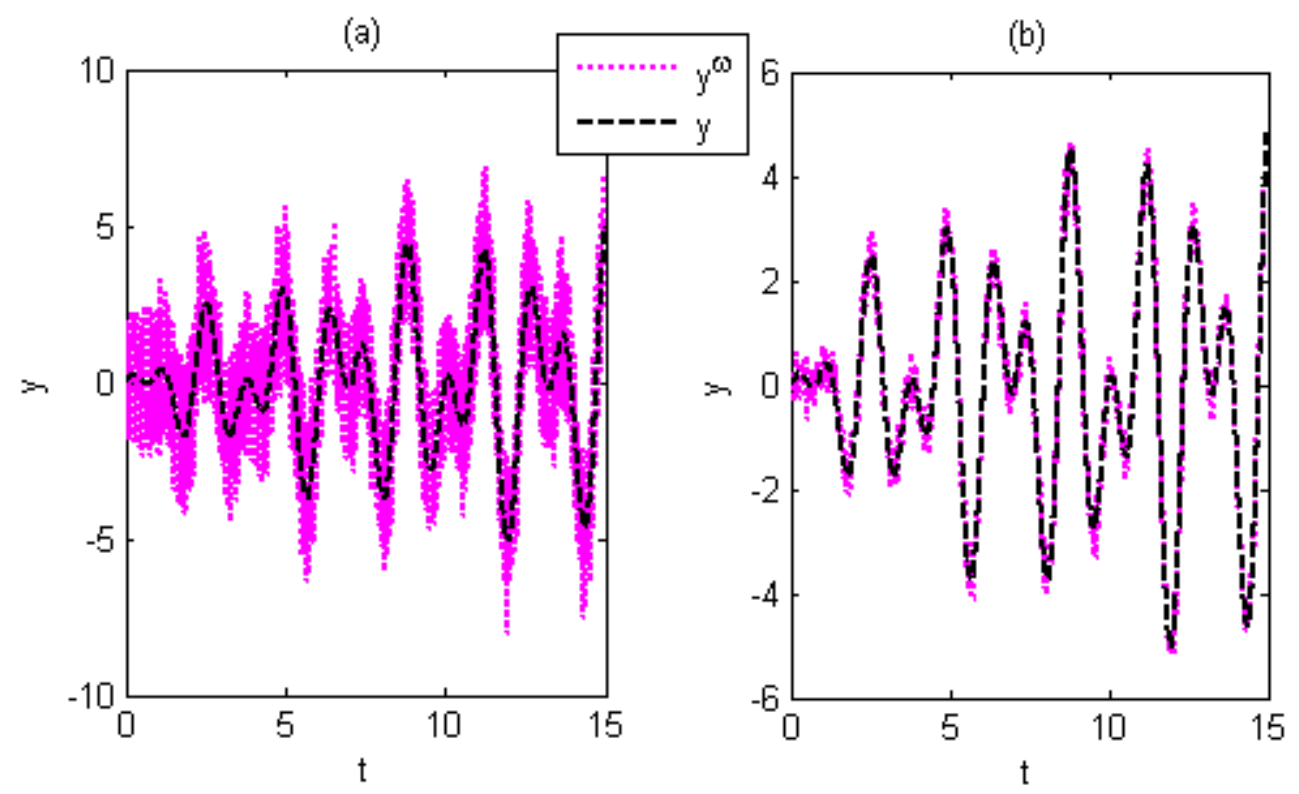

Fig 4.5 Denoising result of the signal in Example 4.3 


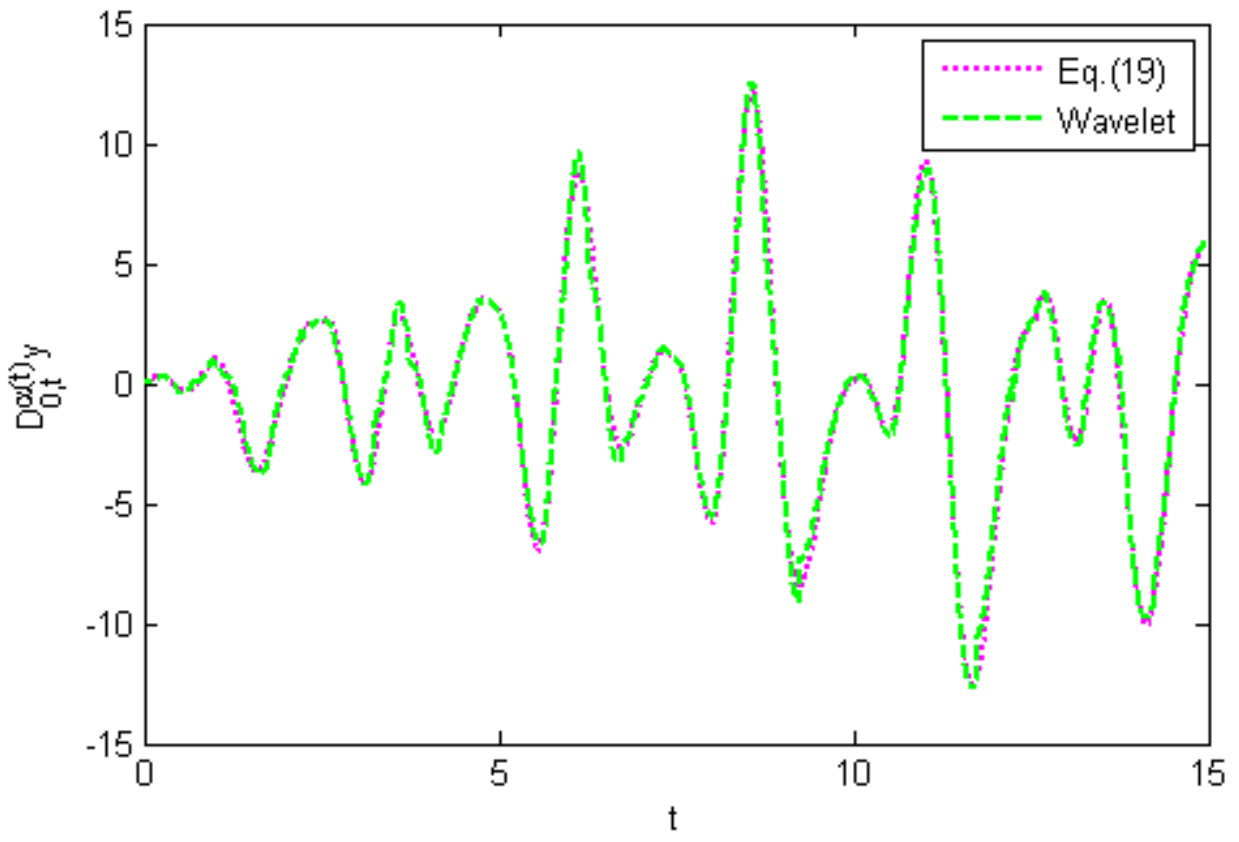

Fig 4.6 The estimated variable-order fractional derivative of Example 4.3

Fig 4.5(a) and the denoised signal is shown in Fig 4.5 (b). The estimated variable-order fractional derivative based on Eq. (18) is depicted in Fig 4.6. Take $\varepsilon=0.01$ in Eq. (20), the corresponding result between Eq. (19) and the wavelet method is 0.1823 . Accordingly, the wavelet method shows better robustness.

Example 4.4 As a special case of the variable-order fractional numerical differentiation, we reset the relative parameters of the considered signal $y(t)=\sin (w t)$ as follows:

$$
w=10, I=[0,4], m=2001, \delta=0.5, \alpha(t)=0.5 .
$$

The signal-to-noise ratio is equal to $S N R=4.95 d B$. The subintervals of $I$ have a length of 1. In [23], the authors present a method combining B-Spline functions and Tikhonov regularization to estimate the Riemann-Lowville fractional derivative of a noisy signal. By using a similar way, the Caputo fractional derivative can also be estimated. Taking $\varepsilon=0.05$ for Eq. (20), the corresponding results of the B-Spline method and the wavelet method are 0.0457 and 0.0280 , respectively. Thus, the wavelet method provides a better result. The Comparison on numerical error of two methods in $I^{\varepsilon}=[0.05,3.95]$ is depicted in Fig 4.7.

According to the above four numerical examples, the validity, robustness and efficiency of the proposed method are clearly verified in four different cases. The wavelet denoising efficiently reduces the noise effect of a signal and the computation of the proposed wavelet method provides satisfactory results as well. Moreover, the last numerical example is presented as a special case of the considered problem to compare with the method based on B-Spline functions. Accordingly, it can be concluded that the proposed wavelet method performs better and the algorithm is also easier to be accomplished. 


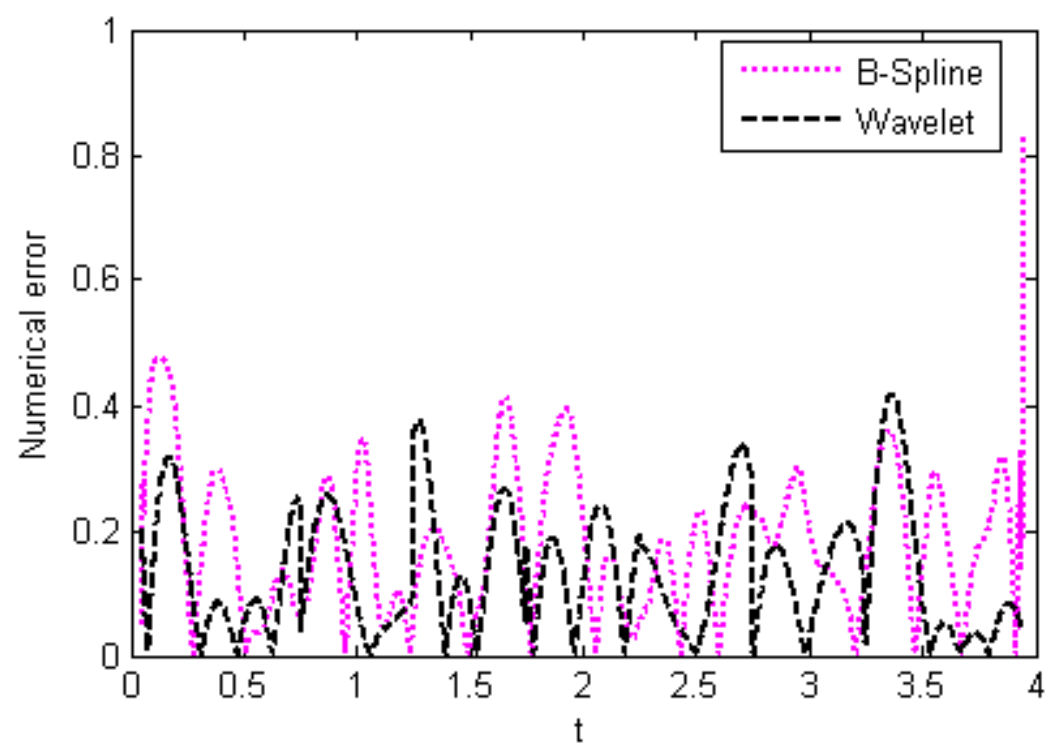

Fig 4.7 Comparison on numerical errors of two methods

\section{Conclusion}

In this paper, a new method based on wavelet denoising is proposed to estimate the variable-order fractional derivatives of noisy signals. The wavelet method is adopted to reduce the noise effect of a signal to a certain degree. Then, the denoised signal is fitted by polynomials in a sequence of overlapped subintervals, where the values obtained near the boundaries of each subinterval are ignored in the overlapped parts. Numerical examples are presented to demonstrate the efficiency and robustness of the proposed wavelet method. It can also be illustrated by numerical examples that the proposed wavelet method shows better performance than the B-Spline method presented in [23] in the case where the fractional differential order is a constant.

\section{Acknowledgement}

This work is supported by the Natural Science Foundation of Heibei Province (A2012203407).

\section{References}

[1] R.L. Bagley, P.J. Torvik, Fractional calculus in the transient analysis of viscoelastically damped structures. AIAA J. 23 (1985) 918-925.

[2] X. Zheng, W. Chen, A fractional-order model on new experiments of linear viscoelastic creep of Hami Melon, Comput. Math. Appl. 66 (2013) 677-681.

[3] R.L. Baillie, Long memory processes and fractional integration in econometrics, J. Econom. 73 (1996) 5-59.

[4] F. Mainardi, Fractional calculus: 'some basic problems in continuum and statistical mechanics', in: A. Carpinteri, F. Mainardi (Eds.), Fractals and Fractional Calculus in Continuum Mechanics, Springer Verlag, New York, 1997, pp. 291-348. 
[5] Y.A. Rossikhin, M.V. Shitikova, Applications of fractional calculus to dynamic problems of linear and nonlinear hereditary mechanics of solids, Appl. Mech. Rev. 50 (1997) 15-67.

[6] W.H. Deng, S.D. Du, Y.J. W, High order finite difference WENO schemes for fractional differential equations, Appl. Math. Lett. 26 (2013) 362-366.

[7] S. Gupta, D. Kumar, J. Singh, Numerical study for systems of fractional differential equations via Laplace transforms, Journal of the Egyptian Mathematical Society. 23 (2015) 256-262.

[8] Momani, Al-Khaled K, Numerical solutions for systems of fractional differential equations by the decomposition method, Appl. Math. Comput. 162 (2005) 1351-1365.

[9] Z. Odibat, S. Momani, Application of variational iteration method to nonlinear differential equations of fractional order, Int. J. Nonlinear Sci. Numer. Simul. 7 (2006) 27-34.

[10] A. Arikoglu, I. Ozkol, Solution of fractional integro-differential equations by using fractional differential transform method, Chaos. Soliton. Fract. 40 (2009) 521-529.

[11] M. Li, W. Zhao, Solving Abel's type integral equation with Mikusinski's operator of fractional order, Advances in Mathematics Physics. Vol. 2013, Article ID 806984, 4 pages, 2013.

[12] G. Bengochea, Operational solution of fractional differential equations, Appl. Math. Lett. 32 (2014) 48-52.

[13] M.X. Yi, J. Huang, J.X. Wei, Block pulse operational matrix method for solving fractional partial differential equation, Appl. Math. Comput. 221 (2013) 121-131.

[14] A. Saadatmandi, Bernstein operational matrix of fractional derivatives and its applications, Appl. Math. Model. 38 (2014) 1365-1372.

[15] Y.M. Chen, Y.N. Sun, L.Q. Liu, Numerical solution of fractional partial differential equations with variable coefficients using generalized fractional-order Legendre functions, Appl. Math. Comput. 244 (2014) 847-858.

[16] L. Zhu, Q.B. Fan, Solving fractional nonlinear Fredholm integro-differential equations by the second kind Chebyshev wavelet, Commun. Nonlinear Sci. Numer Simulat. 17 (2012) 2333-2341.

[17] Y.M. Chen, X.H. Ke, Y.Q. Wei, Numerical algorithm to solve system of nonlinear fractional differential equations based on wavelets method and the error analysis, Appl. Math. Comput. 251 (2015) 475-488.

[18] B. Mathieu, P. Melchior, A. Oustaloup, C. Ceyral, Fractional differentiation for edge detection, Signal Process. 83 (2003) 2421-2432.

[19] M. Benmalek, A. Charef, Digital fractional order operators for R-wave detection in electrocar-diogram signal, IET Signal Process. 3 (2009) 381-391.

[20] D.L. Chen, Y.Q. Chen, D.Y. Xue, Digital Fractional Order Savitzky-Golay Differentiator, IEEE T Circuits-II. 58 (2011) 758-762.

[21] D.Y. Liu, O. Gibaru, W. Perruquetti, T.M. Laleg-Kirati, Fractional order differentiation by integration with Jacobi polynomials, in Proc. 51st IEEE Conference on Decision and Control, Hawaii, USA, 2012.

[22] D.Y. Liu, O. Gibaru, W. Perruquetti, T.M. Laleg-Kirati, Fractional order differentiation by integration and error analysis in noisy environment. IEEE $\mathrm{T}$ Automat Contr, DOI:10.1109/TAC.2015.2417852, 2015.

[23] D.Y. Liu, T.M. Laleg-Kirati, O. Gibaru, W. Perruquetti, Fractional order numerical differentiation with B-Spline functions, in the International Conference on Fractional Signals and Systems 2013.

[24] D.Y. Liu, T.M. Laleg-Kirati, Robust fractional order differentiators using generalized modulating functions method, Signal Process. 107 (2015) 395-406. 
[25] D.Y. Liu, Y. Tian, D. Boutat, T.M. Laleg-Kirati. An algebraic fractional order differentiator for a class of signal satisfying a linear differential equation, Signal Process. 116 (2015) 78-90.

[26] Y.L. Li, C. Pan, X. Meng, Y. Ding, H. Chen, A method of approximate fractional order differentiation with noise immunity, Chemometr. Intell Lab. 144 (2015) 31-38.

[27] K.Y. Zheng, X. Zhang, P.J. Tong, Y. Yao, Y.P. Du, Pretreating near infrared spectra with fractional order Savitzky-Golay differentiation (FOSGD), Chinise Chem Lett. 26 (2015) 293-296.

[28] H.G. Sun, Y. Zhang, W. Chen, D.M. Reeves, Use of a variable-index fractional-derivative model to capture transient dispersion in heterogeneous media, J. Contam Hydrol. 157 (2014) 47-58.

[29] H.G. Sun, W. Chen, H. Sheng, Y.Q. Chen, On mean square displacement behaviors of anomalous diffusions with variable and random orders, Phys. Lett. A. 374 (2010) 906-910.

[30] H.G. Sun, W. Chen, Y.Q. Chen, Variable-order fractional differential operators in anomalous modeling, Physica A. 388 (2009) 4586-4592.

[31] Z.J. Fu, W. Chen, L. Ling, Method of approximate particular solutions for constant- and variable-order fractional diffusion models, Eng. Anal. Boundary Elem.57 (2015) 37-46.

[32] S. Shen, F. Liu, J. Chen, I. Turner, V. Anh, Numerical techniques for the variable order time fractional diffusion equation, Appl. Math. Comput. 218 (2012) 10861-10870.

[33] Y.M. Chen, L.Q. Liu, B.F. Li, Y.N. Sun, Numerical solution for the variable order linear cable equation with Bernstein polynomials, Appl. Math. Comput. 238 (2014) 329-341.

[34] Y.M. Chen, L.Q. Liu, X. Li, Y.N. Sun, Numerical solution for the Variable Order Time Fractional Diffusion Equation with Bernstein Polynomials, CMES: Computer Modeling in Engineering \& Sciences. 97 (2014) 81-100.

[35] Y.M. Chen, Y.Q. Wei, D.Y. Liu, H. Y, Numerical solution for a class of nonlinear variable order fractional differential equations with Legendre wavelets, Appl. Math. Lett. 46 (2015) 83-88.

[36] M. Zayernouri, G.E. Karniadakis, Fractional spectral collocation methods for linear and nonlinear Variable order FPDEs, J. Comput Phys. 293 (2015) 312-338.

[37] X. Zhao, Z.Z. Sun, G.E. Karniadakis, Second-order approximations for variable order fractional derivarives: Algorithms and applications, J. Comput Phys. 293 (2015) 184-200.

[38] S. Mallat, A Wavelet Tour of Signal Processing: The Sparse Way, third ed., Elsevier, Singapore, 2012.

[39] S. Mallat, An efficient image representation for multiscale analysis, Proc. Machine Vision Conference, February, 1987.

[40] S. Mallat, A theory for multiresolution signal decomposition: The wavelet representation, IEEE Trans. Patt. Anal. Mach. Intell., 11 (1989) 674-693. 